\title{
Metodologías activas para la enseñanza de habilidades comunicativas en el grado de medicina
}

\author{
Sonia Ruiz de Azua ${ }^{\text {a }}$, Naiara Ozamiz Etxebarria ${ }^{\text {b }}$, Virginia Guillen ${ }^{c}$, Miren Agurtzane \\ Ortiz Jaureguid, \\ ${ }^{a}$ Universidad del País Vasco. CIBERSAM sonia.ruizdeazua@ehu.eus, ${ }^{b}$ Universidad del País Vasco. \\ naiara.ozamiz@ehu.eus, ${ }^{\mathrm{c}}$ Universidad del País Vasco. Virginia.guillen@ehu.eus ${ }^{\mathrm{d}}$ Universidad del \\ País Vasco.mirenagurtzane.ortiz@ehu.eus
}

\begin{abstract}
Active methodologies are effective for teaching in the university environment and produce a greater and more lasting. Social skills and communications are essential in the health field to improve the doctor-patient relationship, offering a good environment and improving clinical care and satisfaction with the treatment received. In this work, active methodologies have been applied in the teaching of social and communicative skills of the degree of medicine. Various pedagogical techniques have been used as cooperative learning techniques $(1,2,4)$, puzzle, case-based learning, flipped classroom, roleplaying, etc ... After these nine practical sessions you have more ability to face a patient
\end{abstract}

Keywords: social skills, communication, medicine, active learning.

\section{Resumen}

Las metodologías activas son efectivas para la docencia en el ámbito universitario y producen un mayor y más duradero aprendizaje ya que se le hace al propio alumno el dueño de su propio aprendizaje. Las habilidades sociales y comunicativas son esenciales en el ámbito sanitario para mejorar la relación terapéutica médico-paciente, ofreciendo un buen ambiente y mejorando la asistencia clínica y la satisfacción con el trato recibido. En este trabajo se han aplicado las metodologías activas en la enseñanza de las habilidades sociales y comunicativas del grado de medicina. Se han usado diversas técnicas pedagógicas como técnicas de aprendizaje cooperativo $(1,2,4)$, puzzle, aprendizaje basado en casos, flipped classroom, roleplaying, etc... Un alto porcentaje del alumnado considera estas técnicas útiles para el aprendizaje de estas habilidades y opina que después de estas nueve sesiones prácticas tiene más capacidad para enfrentarse a un paciente.

Palabras clave: habilidades sociales, comunicación, medicina, metodologías activas 


\section{Introducción}

\subsection{Qué son las metodologías activas}

Las metodologías activas no es un concepto novedoso, a lo largo de la historia se puede observar cómo tanto autores como instituciones han usado esta denominación para investigar sobre técnicas docentes. Estas metodologías incluyen técnicas y estrategias que fomentan la participación activa del alumno en su formación, haciéndole construir su propio aprendizaje, de tal forma que se aprenda de manera integral y flexible (Labrador y Andreu, 2008).

El plan estratégico de la Universidad del País Vasco apuesta por el modelo educativo denominado Aprendizaje Cooperativo y Dinámico (IKD, del euskera Ikaskuntza Kooperatibo eta Dinamikoa), en el cual se fomenta la utilización de las metodologías activas y en el que adquiere especial relevancia la responsabilidad individual unida a la interdependencia positiva, las habilidades sociales, la interacción directa y la reflexión en grupo. Otros de los puntos que se ha propuesto impulsar es la formación del profesorado impartiendo cursos gratuitos de forma asidua en sus tres campus, accediendo así al profesorado de diferentes grados.

Entre otras técnicas el Aprendizaje Basado en Problemas (ABP) centra la participación del estudiante integrando la parte teórica con la práctica, despertando la curiosidad, creatividad y razonamiento crítico e involucrándole en la búsqueda de la información para su propio aprendizaje. Este método habitualmente en la enseñanza de grados sanitarios, especialmente para el aprendizaje de diversas especialidades de la medicina (Mistry et al., 2019; Lepiller et al., 2017; Sobocan et al., 2017; Bosch-Barrera et al., 2015; Heru 2011)

El de Aprendizaje Basado en Casos es un método que favorece el aprendizaje por descubrimiento, favorece la realización de preguntas y la respuesta a las mismas para la deducción de las conclusiones, favorece la discusión y acerca el mundo académico al mundo profesional. Tanto en las personas que se están formando como en el desarrollo profesional este tipo de metodología mejora las competencias a través del razonamiento lógico, organización, búsqueda de información, análisis y evaluación de datos. Este tipo de metodologías se han aplicado con éxito a los campos sanitarios (Struck and Teasdale, 2008; Scott et al., 2016) observando un mayor aprendizaje en combinación con los mapas conceptuales (Khosa et al., 2014)

El Aprendizaje Cooperativo es una forma de trabajar en grupos reducidos en la que cada parte del grupo maneja determinada información para poder complementarse la información trabajando la capacidad de comunicación y la confianza entre todos los miembros del equipo. Con esta forma de aprendizaje se fomenta el trabajo en equipo para aumentar la productividad y así que todo el grupo salga beneficiado. Son numerosas y diversas las técnicas que aplican el aprendizaje cooperativo: Jigsaw Puzzle, técnica 124, 4 sabios, mapas conceptuales, etc... resultando dinámicas y muy didáctica (Zhang and Cui, 2018)

El flipped classroom es un modelo pedagógico que consiste en trasladar el trabajo de clase sobre determinadas tareas en las que los procesos cognitivos a trabajar son más básicos 
como la lectura o asimilación de la información fuera del aula y anterior a la clase de tal forma que el tiempo dentro del aula se pueda dedicar a tareas más complicadas como son el análisis crítico, el razonamiento, la discusión, etc... Este modelo permite que el docente tenga un feedback sobre lo comprendido por el alumnado anterior a la clase de tal forma que puede estructurar el tiempo de la clase en función de los conocimientos previos e inquietudes de los y las estudiantes. La experiencia de esta metodología en la enseñanza de grados sanitarios ha sido muy positiva mejorando el conocimiento y aportando enriquecimiento a las clases (Graham et al., 2019; French et al., 2018; Komarraju et al., 2018; Tolks et al., 2016)

La simulación o "role playing" es un tipo de técnica pedagógica que parte de la experiencia y de la reflexión que ésta produce en uno mismo. Es una actividad que copia una situación real y tiene un final abierto, no existiendo unas respuestas más correctas que o otras (Jones, 1995). Esta práctica docente permite adquirir habilidades y destrezas que fomentan el pensamiento crítico, la resolución de problemas, la curiosidad intelectual, el pensamiento lógico y la competencia comunicativa (Andreu y García, 2002). Las ventajas del uso de estas técnicas son que integra la parte teórica con la práctica, motivando al alumnado, ofreciéndole un papel activo en su propio aprendizaje y preparándole para el mundo profesional (Ballatore, 2011). Este método permite usar diferentes variantes, como son el caldeamiento, el soliloquio, el doblaje, el espejo o el cambio de roles para sacar un mayor rendimiento (Baile y Blatner, 2014), aportando un aprendizaje experiencial en los estudiantes de ciencias de la salud (Nestel y Tierney, 2007). Así mismo, se ha demostrado que para que el role playing produzca un aprendizaje efectivo es necesario contar con tiempo suficiente para ello así como que se produzca un feedback y una reflexión sobre lo que ha ocurrido (Joyner y Young, 2006).

La evaluación por pares se ha usado en la enseñanza aunque siempre ha provocado controversias. Ha resultado ser efectivo tanto para el aprendizaje de los estudiantes que dan el feedback como para los que lo reciben (Tooping, 2005), e incluso en algunos estudios se encuentran que este tipo de retroalimentación es más relevante que la del propio docente (English et al., 2006). Sin embargo, las críticas a esta forma de evaluación aluden a la insuficiente preparación de los compañeros que dan el feedback e incluso al miedo sobre dar una opinión sincera a un compañero al cual se le tiene aprecio (Wen y Tsai, 2006).

\subsection{Enseñanza de las habilidades sociales y comunicativas en medicina}

La comunicación tanto verbal como no verbal entre el profesional sanitario y el paciente es una parte fundamental dentro del tratamiento en el área de la salud, incluido el campo de la odontología, mejorando la satisfacción del paciente, la adherencia y reduciendo la ansiedad y las quejas de los pacientes (Levinson et al., 1997; Haak et al., 2008). Las habilidades sociales y comunicativas son herramientas muy útiles para los profesionales sanitarios ya que es algo que van a tener que poner en práctica constantemente durante su trabajo habitual. Unas adecuadas habilidades sociales favorecerán la relación clínica sanitariopaciente proporcionando tanto al profesional sanitario como al paciente satisfacción por haber recibido un buen trato (Tongue et al., 2005). Una comunicación adecuada entre terapeuta y paciente es un instrumento esencial en el abordaje de la enfermedad e influye 
directamente en el cumplimiento terapéutico, la prevención de conflictos, la mejora de los resultados clínicos y la eficiencia de los servicios sanitarios (Howick, et al., 2018). Además, un buen ambiente y comunicación con los compañeros de trabajo reduce el estrés y las tensiones (Pades, 2002) y esto es realmente importante en una profesión donde es tan frecuente el bournout (Penberthy et al., 2018).

Las habilidades comunicativas es algo que puede estar innato en el individuo, variando esta capacidad innata de unas personas a otras, sin embargo, es posible aprenderlas. Las investigaciones sugieren que las habilidades comunicativas no mejoran necesariamente con la experiencia, sino que cuando hay un déficit es necesario formarse. Este tipo de habilidades no siempre se adquieren durante la formación sanitaria estando muy poco presentes en el currículo de los grados a pesar de ser competencias básicas para un correcto desempeño de la práctica clínica.

La necesidad de integrar las habilidades de comunicación en las enseñanzas de ciencias de la salud está reconocido desde hace años, tanto de forma transversal, como incluyendo créditos específicos sobre la materia dentro del grado, no obstante, no siempre se recoge en todos los grados de medicina, ni se trabajan de forma transversales en las asignaturas clínicas (Cláries, Borrell, 2003). De esta forma el objetivo de la docencia en habilidades para comunicar debería estar presente en todos los niveles educativos del grado tanto de forma transversal como de manera específica, pero no sólo dejarlo ahí, sino que es necesario seguir abordándose como formación profesional continuada durante toda la práctica clínica.

Se están confeccionando diversos cursos y talleres cuyo objetivo es el entrenamiento en habilidades sociales y comunicativa en diversas especialidades clínicas, sobre todo en atención primaria y en especialidades en las que es necesario habilidades específicas comunicativas, como puede ser oncología o pediatría, en los que es tan necesario mantener una buena alianza terapéutica (Fallowfield 2001; Fellowes, 2008). Profesionales de la salud asisten a cursos y talleres de habilidades comunicativas para mejorar la atención a sus pacientes, con el objetivo especifico mejorar el conocimiento de las preocupaciones y sentimientos del usuario que acude al sistema sanitario y posibilitar una atención óptima (Fellowes, 2008). Cada vez son más los programas para la mejora de las habilidades comunicativas en médicos que se dedican al manejo de enfermedades con pronóstico grave como es el cáncer (Fellowes, et al., 2004; Gysel, et al., 2004). Éstos están centrados en la comunicación de malas noticias y en la organización de los objetivos del tratamiento (Back, et al., 2007).

La mayoría de los enfoques de enseñanza incorporan componentes cognitivos, afectivos y conductuales, y se centran en el sujeto que está aprendiendo, en el sentido de "llevar las riendas" del diálogo o entrevista en un marco de atención clínica. Algunas revisiones sitematicas se centran la efectividad de las actividades para mejorar estas competencias (Omura et al. 2017), mientras que otras se centran en las habilidades a desarrollar por el dinamizador en función de sus atributos clínicos, habilidades de enseñanza y cualidades personales relacionadas con el desarrollo de habilidades comunicacionales (Burguess et al. 2015). 
La evaluación de habilidades de comunicación tiene su complicación ya que un examen tradicional no reúne las competencias suficientes para que demuestren que han obtenido los resultados de aprendizaje necesarios para la superación de la asignatura. Es por ello por lo que debe realizarse a través de metodologías que aúnen el trabajo con casos y situaciones lo más reales posibles, creando escenarios y personajes cercanos a la realidad y con la utilización de recursos teatrales que le aporten veracidad, en un marco de práctica reflexiva, análisis del discurso, aprendizaje significativo y metacomunicación (Fernandez et al., 2010). La coevaluación, evaluación colaborativa entre alumnos y profesores, y la evaluación entre iguales o por pares (Brawn y Glasner, 2007; Dochy, 2006) son estrategias de evaluación participativa y formativa, con numerosos beneficios descritos en la bibliografía educativa e implica una concepción más democrática del proceso de evaluación.

\section{Objetivos}

El objetivo de este trabajo es el análisis de la metodología pedagógica que se usa para enseñar las habilidades sociales y comunicativas en la asignatura de Comunicación y Relación Clínica. Con ello se pretende que los alumnos se impliquen en su propio aprendizaje tomando esta competencia la importancia que requiere.

Los objetivos específicos de este trabajo:

- Analizar cuáles son las habilidades sociales y comunicativas claves para iniciar a los estudiantes de medicina en formación en estas habilidades.

- Introducir las metodologías activas en éste área de conocimiento.

- Conocer la satisfacción y efectividad de estas metodologías en el aprendizaje de estas habilidades.

- Evaluar la mejora en estas habilidades de los alumnos de $3^{\text {er }}$ curso del grado de medicina.

\section{Desarrollo de la innovación}

Esta innovación educativa se realiza durante la asignatura de Ética, Comunicación y Relación Clínica impartida en el $3^{\text {er }}$ curso del grado de medicina. Esta asignatura se compone de 6 créditos ECTS de los cuales 3 corresponde a la parte de Comunicación y relación clínica. Éstos están formados por 11 horas de clase magistral, 9 prácticas de aulas de 2 horas de duración y 3 seminarios de dos horas.

Las competencias y resultados de aprendizaje de esta parte de la asignatura son están relacionados con el desarrollo de las habilidades sociales como empatía y escucha activa, así como el aprendizaje de habilidades comunicativas que permitan conocer y analizar los estilos comunicativos así como relacionarse con los pacientes y con los propios compañeros.

Generalmente la metodología elegida por el alumnado es la evaluación continua en la que el examen teórico vale un 50\%, la asistencia, participación y los trabajos realizados durante las 9 prácticas de aula vale un $20 \%$ y los seminarios el $30 \%$ restante, durante los cuales se 
pone en práctica a través una escenificación las técnicas y habilidades adquiridas durante las prácticas.

Los contenidos prácticos de la asignatura están enfocados en trabajar las siguientes habilidades sociales y comunicativas:

- Escucha activa y empatía

- Habilidades sociales y estilos comunicativos

- Relación médico-paciente

- Entrevista clínica

- Entrevista clínica II

- Como dar malas noticias

- Técnicas de negociación y resolución de conflictos

- Técnicas de comunicación en el duelo y en situaciones difíciles

\subsection{Variables.}

Para la evaluación de las habilidades sociales se han utilizado cuatro test diferentes que evalúan respectivamente la escucha activa (Test de Escucha Activa), la empatía (Test de Empatía Cognitiva y Afectiva (TECA), la asertividad (Autoinforme de Conductas Asertivas (ADCA-1) y otras habilidades sociales (Escala de Habilidades Sociales (EHS). El tiempo estimado de realización de los test fue de 20 minutos. Esta encuesta se pasa antes de las clases para conocer cuáles son sus habilidades sociales y comunicativas en el contexto de las clases de la asignatura de comunicación y relación clínica, por lo que la respuesta a la realización de este ejercicio es muy amplia. Posteriormente, y una vez finalizada la asignatura se vuelve a dar la opción a los y las estudiantes de volver a realizar los 4 test.

El Test de Escucha Activa es una prueba no validada, compuesta de 20 ítems, con dos opciones de respuesta (sí y no) y cuatro escalas: escuchar sin interrumpir, escuchar prestando el $100 \%$ de atención, escuchar más allá de las palabras, y escuchar incentivando al otro a profundizar.

El test TECA o Test de Empatía Cognitiva y Afectiva, (López-Pérez et al., 2008) fue creado como instrumento para medir la empatía. Está formado por 33 elementos medidos mediante la escala Likert de respuesta (totalmente en desacuerdo, algo en desacuerdo, neutro, algo de acuerdo y totalmente de acuerdo). Consta de cuatro escalas de evaluación; adopción de perspectivas, la comprensión emocional, y la capacidad de identificar los estados emocionales negativos (estrés empático), así como la capacidad de identificar las emociones positivas (alegría empática).

El Test ADCA-1 o Autoinforme de Conducta Asertivas, se publicó originalmente en el año 1992 (García Pérez \& Magaz Lago, 1992) de manera independiente, pero ahora forma parte de los Autoinformes ADCAS. Consta de dos partes, una con 20 ítems y otra de 15 ítems, con cuatro opciones de respuesta (nunca o casi nunca, a veces, a menudo y siempre o casi siempre). Estas dos partes, evalúan respectivamente la auto asertividad (el respeto y compromiso con los pensamientos y deseos propios), y la hetero asertividad (asertividad hacia los deseos ajenos). Por otro lado, permite identificar el estilo personal en las habilidades sociales: agresivo, pasivo y asertivo. 
Por último, usamos el test EHS de Estudio de las Habilidades Sociales (Gismero González, 2000). Consta de 33 ítems; 28 redactados en sentido negativo, de falta de aserción o déficit de habilidades sociales, y 5 en sentido positivo con 4 opciones de respuesta (no me identifico, no tiene que ver conmigo, me describe aproximadamente y estoy muy de acuerdo). Evalúa la capacidad asertiva en situaciones sociales, la defensa de los derechos como consumidor, la expresión de enfado o disconformidad, saber decir que no, la capacidad de hacer peticiones y las interacciones con el sexo opuesto.

Se aseguró el anonimato asignando un código a cada estudiante que consta de 4 letras: inicial de la madre, día de nacimiento de la madre, inicial del padre y día de nacimiento del padre. Este código, además de asegurar el anonimato garantizó que los estudiantes no lo olvidaran. Los alumnos de tercer curso realizaron el test en el aula en el contexto de la asignatura Comunicación y Relación Clínica durante el mes de marzo.

Este estudio ha pasado por el Comité de Ética para la Investigación con Seres Humanos, CEISH-UPV/EHU, BOPV32,17/02/2014, con el código M10_2018_263, para la realización del proyecto de investigación: "Estudio sobre la capacidad de escucha activa, empatía, asertividad y habilidades sociales" recibiendo el informe favorable el 20 de febrero de 2019.

Por otro lado, al final del curso y una vez realizado el examen se pasó una encuesta para conocer la opinión del alumnado sobre los contenidos de la asignatura, el empleo de las técnicas y su autopercepción sobre el aprendizaje y mejora de estas habilidades (anexo 1).

\subsection{Participantes}

La muestra estuvo conformada por 300 estudiantes de tercer curso del Grado en Medicina de la Universidad del País Vasco, Bizkaia, España. De los participantes el 75,8\% eran mujeres $(75.8 \%)$ y el $23.5 \%$ varones. Sus edades oscilaban entre los 19 y 43 años, resultando un promedio de edad de 21.5 años y una mediana de 20 años. La participación en el estudio fue voluntaria y se comunicó a los participantes que al finalizar la investigación recibirían información en profundidad sobre los resultados del estudio.

\subsection{Implementación en la clase mediante las metodologías activas.}

Se parte de un caso clínico en el que aparecen 4 personajes por lo cual permite ver diferentes visiones en función del personaje desde el que se observa. El protagonista es un paciente con cáncer en tratamiento cuyos efectos secundarios le están siendo molestos y con síntomas depresivos los cuales le hacen pensar en tirar la toalla y no seguir con el tratamiento. Su mujer, persona ansiosa e invasiva que no tiene asumida la enfermedad de su marido y no le escucha y respeta. La oncóloga, persona asertiva con gran capacidad de empatía, escucha en todo el momento al paciente y respetuosa con sus decisiones ya que pasó un cáncer hace años. Y el residente de tercer año que está haciendo una tesis doctoral en los nuevos tratamientos de cáncer, el cual tuvo una mala experiencia con esta enfermedad ya que su madre murió cuando él era pequeño. 
A través de este caso y usando diversas metodologías activas se irán viendo las diferentes técnicas comunicativas y sociales para llegar a un guion final en el que se pueda realizar una escena lo más óptima posible.

- TECNICA COOPERATIVA 1,2,4 : después de leer el caso cada estudiante relee de nuevo el caso y subraya las palabras claves con respecto a las habilidades sociales y comunicativas necesarias poner en práctica para resolver este caso. Se pone en común en parejas. Se pone en común en grupos de 4 y posteriormente se pone en común en el grupo grande y se escribe en la pizarra de papel. Entre otras aparecerán:

- Decir una mala noticia

- Asertividad

- Negociación

- Motivar y persuadir

- Salto psicosocial

- Entrevista básica

- Pacientes y familiars difíciles

- TÉCNICA PUZZLE: se hacen grupos pequeños de 5-7 personas y se les entrega un artículo que tienen que leer analizar y resumir. Posteriormente se vuelven hacer grupos en el que haya un representante de cada una de las partes de los grupos y tienen que explicarse los unos a los otros en qué consiste cada una de las técnicas que se explican en los textos.

- FLIPCLASSROOM: esta técnica se utiliza para aprovechar el tiempo en el aula por lo que antes de casa clase se sugiere la visión de videos o lecturas cortas. Se les facilita a través de egela los siguientes enlaces para que vean en casa dos videos donde se explica lo que es la escucha activa y la empatía que luego trabajaremos en clase. Así mismo, tienen disponible 3 videos de actrices donde se ejemplifica en el contexto clínico tanto la escucha activa como la empatía. Estos videos fueron grabados por las profesoras que se encargan de la docencia de esta asignatura. Tendrán que contestar a unas preguntas a través del programa Socrative.

- ROLEPLAY: en grupos de 4 personas hacer un diálogo de unos 3 minutos sobre cómo ha podido ser la conversación de la escena que aparece en el caso. Salen varios grupos a realizar el roleplay. Se comenta en grupo cada roleplay. En el roleplay final tienen que realizar la misma escena pero usando toda la información que han aprendido: entrevista, técnicas de escucha activa y empatía, técnicas de negociación de tratamiento, manejo de familiar invasivo, salto psicosocial, decir malas noticias, técnicas asertivas para comunicar, etc...

- TÉCNICA JIGSAW: como en el roleplay ha habido 4 personajes, cada persona del grupo ha podido experimentar lo que siente cada personaje. Ahora se juntan en función del personaje que es cada uno y reflexionan sobre cómo se siente su personaje. Se tienen que centrar en las emociones, dudas, miedos, inseguridades y 
usar alguna técnica asertiva para comunicar a otro personaje como se le hace sentir. Se habla desde la primera persona.

- TÉCNICA DE LOS SOMBREROS: Cada grupo de personaje habla sobre cómo se ha sentido, se habla desde la primera persona y se dirige a otro personaje desde la segunda persona.

- ENTREGABLES: a través de la plataforma moodle se enviará el guión inicial y final del caso así como un informe sobre las emociones de cada personaje.

\subsection{Análisis estadísticos}

Para el análisis de datos se ha usado el programa estadístico SPSS 23. Se ha realizado una base de datos con las variables de los 4 test así como con la información demográfica que el alumnado de medicina ha contestado. Se han realizado frecuencias y descriptivos de la muestra así como se ha analizado la homogeneidad de la misma. Para análisis de variables cualitativas se ha usado el estadístico Chi cuadrado (X2). Se han usado pruebas no paramétricas para muestras relacionadas para evaluar el cambio en las competencias sociales del los test al inicio de las prácticas y al final. D

\section{Resultados}

El alumnado del grado de medicina posee unas buenas habilidades en la escala de escucha activa, siendo la escala de escuchar incentivando a profundizar en la que mayor puntuación obtiene, necesitando trabajar la capacidad de atención en las palabras del paciente. La puntuaciones obtenidas en la capacidad empática de los estudiantes era media alta tanto en adopción de perspectivas como en la comprensión emocional o alegría empática, no obstante, era menor su capacidad para identificar los estados emocionales negativos. Los valores obtenidos en el test EHS son medios estando comprendidos entre 40 y 60 en las escalas de capacidad asertiva en situaciones sociales, defensa de los derechos como consumidor, la expresión de enfado o disconformidad, saber decir que no y las interacciones con el sexo opuesto, siendo la capacidad de hacer peticiones la escala en la que menor puntuación obtienen.

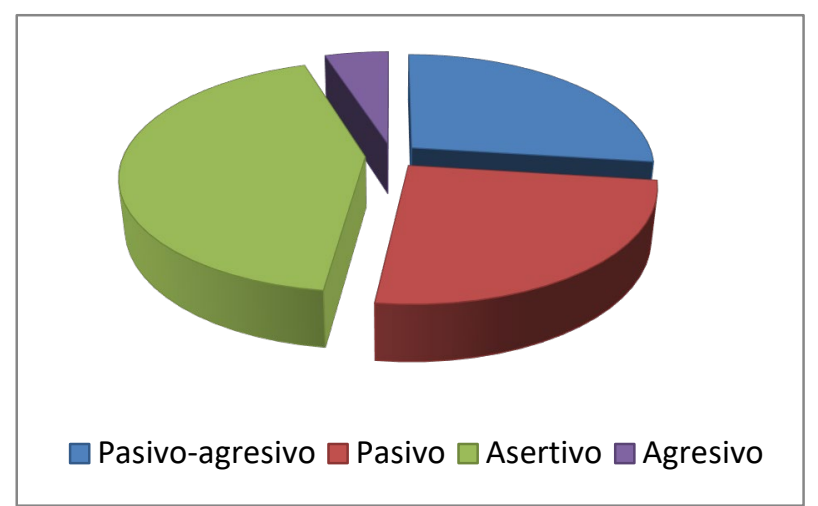

Con respecto a la asertividad el alumnado rozaba valores bajos en autoasertividad y medios en heteroasertividad, dejando un mapa final con respecto al estilo de comunicación del alumnado según la figura 1 : $27 \%$ pasivo agresivo, $25 \%$ pasivo, $43 \%$ asertivo y $5 \%$ agresivo.

Fig. 1 Distribución de estilos de comunicación 
Una vez realizadas las prácticas se volvió a pedir la colaboración del alumnado volviendo a rellenar los test. Los resultados muestran mejoras en todas las escalas del cuestionario de escucha activa. Una mejora la capacidad empática general y, sobre todo, en las escalas de adopción de perspectiva y la capacidad para identificar los estados emocionales negativos. Una mejora en la capacidad para mostrar enfado o disconformidad y en para realizar peticiones. Así mismo, mejora su capacidad de auto asertividad haciendo mejorar su capacidad asertiva.

En cuanto al empleo de las metodologías pedagógicas, las técnicas que más útiles les ha parecido a los alumnos han sido el rolepaying, el trabajo con casos clínicos, el trabajo y discusión en grupos pequeños y el feedback tanto de los propios compañeros como del docente. Al 93,2\% de los estudiantes les parecía adecuado el uso del caso para la enseñanza y aprendizaje de este tipo de competencias, considerando que el diseño del caso era adecuado. El trabajo en grupo también era una metodología bien considerada entre los estudiantes los cuales se habían sentido bien trabajando en diversos grupos y cambiando el rol de trabajo en función del grupo. Los vídeos usados antes de las clases también son considerados adecuados para la pedagogía de las habilidades comunicativas. El flipped classroom no era tan aceptado por los alumnos ya que un 64\% creían adecuado el uso de esta técnica, mientras que un $46 \%$ había echando de menos las explicaciones de la profesora.

El $94,3 \%$ considera el roleplay como una técnica útil para el entrenamiento en las habilidades sociales y comunicativas, sin embargo, el 54,3\% no se sintió cómodo realizando el roleplay delante de toda la clase. El $86,3 \%$ de los estudiantes consideraba que había trabajado de forma activa durante las prácticas y el $94 \%$ consideraba que disponía de mayores estrategias para enfrentarse a un paciente.

Las prácticas que más les han interesados son las de decir malas noticias y empatía, seguida por las técnicas de relación de médico paciente y asertividad. Por otro lado las metodologías que más han gustado son los casos clínicos y el feedback de los roleplays, considerando que esta metodología les permitía ampliar su aprendizaje.

\section{Conclusiones}

Actualmente cada vez son más las universidades que se percatan de la importancia de las competencias comunicativas y que incluyen dentro de su curriculum docente asignaturas específicas que traten estas competencias o lo añaden como competencias transversales a lo largo del grado (Roze des Ordons, 2017).

El diseño de las actividades docentes a través de metodologías activas, está suponiendo una reelaboración de algunos aspectos de la asignatura dentro del grado de medicina, siendo escasas las horas dedicadas a ello. En nuestro caso, hemos decidido utilizar una combinación de técnicas pasivas (magistrales) y activas (prácticas de aula) en las que incluyen técnicas cooperativas, role playings, aprendizaje basado en casos, aula invertida, evaluación de pares, etc... para dar un mayor dinamismo y atractivo en la implementación 
individual y grupal de las actividades docentes. No obstante, no existe un acuerdo sobre cómo se puede enseñar o aprender las habilidades sociales y comunicativas, ni cuáles son las más importantes dentro del campo sanitario no encontrando diferencias significativas entre ellas (Latif et al., 2018) . Por lo tanto, resulta de especial relevancia determinar cuáles son las habilidades comunicacionales más importantes para el trabajo de un médico. Además, es imprescindible conocer si la intervención, bien mediante asignaturas específicas orientadas al aprendizaje o bien incluyéndolas en asignaturas del grado de forma transversal o incluso reforzándolas durante las prácticas clínicas, puede influir en las competencias comunicativas de los alumnos.

Varias de estas técnicas se encuentran entre las más empleadas, como son el role playing, los pacientes simulados y los pacientes reales, en las que se van introduciendo variaciones como las grabaciones de los role playings o el feedback de los compañeros (Baile y Blatner, 2014 ; Nestel y Tierney, 2007; Joyner y Young, 2006). No obstante, existen otras técnicas como el aprendizaje online (Wagner et al., 2011), el paciente como instructor (Broder et al., 2015), el aprendizaje por pares (Krause et al., 2017), y la observación clínica (Ali, 2013) que también se muestran efectivas pero no son tan utilizadas. Por tanto, no se puede establecer una metodología única y más efectiva frente a otras, ya que cada una ejerce su función dentro del aprendizaje. Cada docente deberá escoger la combinación de técnicas que mejor se adecué a su diseño metodológico y a las habilidades comunicativas que crea más adecuadas incluir en su proyecto docente.

Todavía es necesario seguir mejorando tanto la metodología pedagógica usada como los contenidos pero el alumnado ha respondido bien considerando que ha realizado un buen aprendizaje de las habilidades sociales y comunicativas de tal forma que se sienten más preparados para enfrentarse a los pacientes.

Seguiremos analizando nuevas técnicas y probándolas en la docencia de esta asignatura para poder llegar a una óptima docencia y aprovechar los que consideramos que son escasos créditos dedicados a algo tan importante como las competencias sociales y comunicativas en la profesión sanitaria.

Así mismo, contemplamos realizar un seguimiento en años posteriores para conocer cuál es la evolución de estas competencias a lo largo del grado así como la influencia que tiene las prácticas con pacientes reales en la escucha activa, empatía, asertividad y habilidades sociales. 


\section{Referencias}

ALI NK. (2013). Are we training residents to communicate with low health literacy patients? J Community Hosp Intern Med Perspect. Jan 7;2(4).

ANDREU, M ${ }^{\mathrm{a}}$ A. Y GARCÍA, M. (2002). Effective Communication through a Job Hunting Simulation. The International Simulation and Gaming Yearbook, vol. 11 (ISAGA/SAGSET) CDROM.

BACK AL, ARNOLD RM, BAILE WF, et al. Efficacy of communication skills training for giving bad news and discussing transitions to palliative care. Arch Intern Med 2007;167:453-60.

BAILE, W.F., BLATNER, A. (2004) Teaching communication skills: using action methods to enhance role-play in problem-based learning. Simulation in Healthcare, 9(4), pp.220-7.

BALLATORRE, M. (2011). El uso de las simulaciones para la formación. Emergencia del contexto tecnológico. Enfoque. Learning Review. España

BOSCH-BARRERA J, BRICEÑO GARCÍA HC, CAPELLA D, DE CASTRO VILA C, FARRÉS R, QUINTANAS A, RAMIS J, ROCA R, BRUNET J. (2015). Teaching Bioethics to Students of Medicine with Problem-Based Learning (PBL)]. Cuad Bioet; 26(87):303-9.

BURGESS, A.,GOUSLTON, K., OATES, K. (2015) Role modeling of clinical tutors: a focus group study among medical students. BMD Med Educ, 15(17).

CRAVENER PA. (1997) Promoting active learning in large lecture classes. Nurse Educ.22(3):21-6.

CURRY RH, MAKOUL G. (1996). An active-learning approach to basic clinical skills. Acad Med.71(1):41-4.

ENGLiSH, R., BROOKES, S.T., AVERY, K., BLAZEBY, J.M., BEN-SHLOMO, Y. (2006). The effectiveness and reliability of peer-marking in first-year medical students. Medical Education, 40, pp.965-972.

FELLOWES D, WILKINSON S, MOORE P. Communication skills training for health care professionals working with cancer patients, their families and/or carers. Cochrane Database Syst Rev 2004;(2):CD003751.

FERNANDEZ, MA, GONZÁLEZ JL, LÓPEZ I, MANSO ME. (2010) Evaluación participativa en habilidades para comunicar en $3^{\circ}$ de grado de enfermería en el curso 2009/10, la escenificación como método docente y de evaluación. Revista de docencia Universitaria, 8(2) 73-93.

FRENCH H, GRAY M, GILLAM-KRAKAUER M, BONACHEA EM, CARBAJAL M, PAYNE A, VASQUEZ MM, RUBINOS L, FALCK A, IZATT S, DADIZ R. (2018). Flipping the classroom: a national pilot curriculum for physiology in neonatal-perinatal medicine. J Perinatol. ;38(10):14201427.

GARCIA PEREZ, M. E., \& MAGAZ LAGO, Á. (1992). Autoinforme de Conducta Asertiva

(ADCA-1).

GISMERO GONZÁLEZ, E. (2000). Escala de habilidades sociales: manual. TEA Ediciones.

GRAHAM KL, COHEN A, REYNOLDS EE, HUANG GC. (2019). Effect of a Flipped Classroom on Knowledge Acquisition and Retention in an Internal Medicine Residency Program. J Grad Med Educ. Feb;11(1):92-97. 
GYSELS M, RICHARDSON A, HIGGINSON IJ. Communication training for health professionals who care for patients with cancer: a systematic review of effectiveness. Support Care Cancer 2004;12:692-700.

HAAK, R., ROSENBOHM, J., KOERFER, A., OBLIERS, R., WICHT, M.J. (2008). The effect of undergraduate education in communication skills: a randomized controlled clinical trial. European Journal of Dental Education, 12, (4), pp.213-218

HASLETT, L. (1969). McMaster University introduces Problem-Based Learning in Medical Education [en línea]. En Daniel Schugurensky (ed.) History of Education: Selected Moments of the 20th Century, 2001.

HERU AM. (2011).Teaching psychosomatic medicine using problem-based learning and roleplaying. Acad Psychiatry:;35(4):245-248.

HOWICK J, MOSCROP A, MEBIUS A, et al. (2018). Effects of empathic and positive communication in healthcare consultations: a systematic review and meta-analysis. $J R S o c$ Med.;111(7):240-252.

JOHNSON, D. W. Y JOHNSON, R. T. (1987). Learning together and alone. Englewood Cliffs, NJ: Prentice-Hall.

JONES, K. (1995). Simulations. A Handbook for Teachers and Trainers. London: Kogan Page Ltd.

JOYNER B, YOUNG L (2006). Teaching medical students using role play: twelve tips for successful role plays. Med Teach. May;28(3):225-9

KHOSA DK, VOLET SE, BOLTON JR. (2014). Making clinical case-based learning in veterinary medicine visible: analysis of collaborative concept-mapping processes and reflections. J Vet Med Educ. Winter;41(4):406-17

KOMARRAJU A, BARTEL TB, DICKINSON LA, GRANT FD, YARBROUGH TL. (2018). Implementation of a Flipped Classroom for Nuclear Medicine Physician CME. J Contin Educ Health Prof. Summer;38(3):198-204.

LABRADOR MJ, ANDREU MA. (2008) Metodologías Activas. Grupo de innovación en Metodologías Activas (GIMA). Valencia: Editorial UPV.

LATIF R, MUMTAZ S, MUMTAZ R, HUSSAIN A. (2018). A comparison of debate and role play in enhancing critical thinking and communication skills of medical students during problem based learning. Biochem Mol Biol Educ. Jul;46(4):336-342

LEPILLER Q, SOLIS M, VELAY A, GANTNER P, SUEUR C, STOLL-KELLER F, BARTH H, FAFI-KREMER S. (2017). Problem-based learning in laboratory medicine resident education: a satisfaction survey. Ann Biol Clin (Paris).

LEVINSON, W., ROTER, D.L., MUlloOly, J.P., DULl, V.T., FRANKEL R.M. (1997). Physician-patient communication. The relationship with malpractice claims among primary care physicians and surgeons. Journal of the American Medical Association, 277, pp. 553-559.

LOPEZ-PEREZ, B., FERNANDEZ-PINTO, I., \& ABAD, F. J. (2008). TECA, Test de Empatía Cognitiva y Afectiva. TEA Ediciones. 
MISTRY K, CHETTY NC, GURUNG P, LEVELL NJ. (2019). Digital Problem-Based Learning: An Innovative and Efficient Method of Teaching Medicine. J Med Educ Curric Dev.

NESTEL, D., TIERNEY, T. (2007) Role-play for medical students learning about communication: guidelines for maximising benefits. BMC Medical Education , 2; 7, pp. 3.

OMURA M, MAGUIRE J, LEVETT-JONES T, STONE TE. (2017). The effectiveness of assertiveness communication training programs for healthcare professionals and students: A systematic review. Int J Nurs Stud. Nov;76:120-128

PADES A, FERRER VA. (2002). Cómo mejorar las habilidades sociales. Grupo Editorial Universitario..

PENBERTHY JK, CHHABRA D, DUCAR DM, AVITABILE N, LYNCH M, KHANNA S, XU Y, AIT-DAOUD N, SCHORLING J. (2018). Impact of Coping and Communication Skills Program on Physician Burnout, Quality of Life, and Emotional Flooding. Saf Health Work;9(4):381-387.

ROZE DES ORDONS AL, DOIG CJ, COUILLARD P, LORD J. (2017). From Communication Skills to Skillful Communication: A Longitudinal Integrated Curriculum for Critical Care Medicine Fellows. Acad Me ;92(4):501-505

SCOTT BL, BARKER B, ABRAHAM R, WICKLESS HW. (2016). Integration of DermatologyFocused Physical Diagnosis Rounds and Case-Based Learning within the Internal Medicine Medical Student Clerkship. J Med Educ Curric Dev.

SOBOCAN M, TURK N, DINEVSKI D, HOJS R, PECOVNIK BALON B. (2017).Problem-based learning in internal medicine: virtual patients or paper-based problems? Intern Med J.

STRUCK BD1, TEASDALE TA. (2008). Development and evaluation of a longitudinal Case Based Learning (CBL) experience for a geriatric medicine rotation. Gerontol Geriatr Educ.;28(3):105-14.

TOLKS D, SCHÄFER C, RAUPACH T, KRUSE L, SARIKAS A, GERHARDT-SZÉP S, KLLAUER G, LEMOS M, FISCHER MR, EICHNER B, SOSTMANN K, HEGE I. (2016).An Introduction to the Inverted/Flipped Classroom Model in Education and Advanced Training in Medicine and in the Healthcare Professions. GMS J Med Educ. May 17;33(3)

TONGUE J.R., EPPS H.R., FORESE L.L. (2005). Communication skills for patient-centered care: research-based, easily learned techniques for medical interviews that benefit orthopaedic surgeons and their patients. J Bone Joint Surg Am, 87 pp. 652-658.

WEN, M.L., TSAI, C.C. (2006). University students' perceptions of and attitudes toward (online) peer assessment. Higher Education, 51, pp.27-44.

ZHANG J, CUI Q. (2018). Collaborative Learning in Higher Nursing Education: A Systematic Review. J Prof Nurs. ;34(5):378-388. 


\section{ANEXO 1 \\ PREGUNTAS SATISFACCIÓN PRÁCTICAS COMUNICACIÓN}

Puntúa de 0-5 donde 0 es la menor puntuación y 5 la mayor en qué medida te parece útil para el aprendizaje las siguientes metodologías usadas durante estas clases:

\begin{tabular}{|l|l|l|l|l|l|l|l|l|l|l|l|}
\hline TÉCNICAS & $\mathbf{1}$ & $\mathbf{2}$ & $\mathbf{3}$ & $\mathbf{4}$ & $\mathbf{5}$ & PRÁCTICAS & $\mathbf{1}$ & $\mathbf{2}$ & $\mathbf{3}$ & $\mathbf{4}$ & $\mathbf{5}$ \\
\hline Roleplay & & & & & & Escucha activa y empatía & & & & & \\
\hline Discusión grupal (grupo entero) & & & & & & Artículos HHSS y asertividad & & & & & \\
\hline Trabajo en pequeños grupos (4/6) & & & & & & Relación médico-paciente & & & & & \\
\hline Visionado de videos previos & & & & & & Entrevista clínica & & & & & \\
\hline Trabajo con casos clínicos & & & & & & Como dar malas noticias & & & & & \\
\hline Trabajos entregables por escrito & & & & & & Técnicas de negociación & & & & & \\
\hline Exposición de los trabajos & & & & & & Técnicas de comunicación duelo & & & & \\
\hline Feedback de los compañeros & & & & & & Roleplay final & & & & \\
\hline Feedback de la profesora & & & & & & & & &
\end{tabular}

\begin{tabular}{|c|c|c|c|c|}
\hline & & $\mathrm{Si}$ & No & ¿? \\
\hline 1 & $\begin{array}{l}\text { ¿Te ha parecido adecuado caso las habilidades sociales y comunicativas trabajar mediante un } \\
\text { caso? }\end{array}$ & & & \\
\hline 2 & ¿Consideras que ha sido útil la visualización del video antes de la práctica? & & & \\
\hline 3 & $\begin{array}{l}\text { ¿Crees que visualizando la explicación antes de la clase se aprovecha mejor el tiempo de las } \\
\text { prácticas? }\end{array}$ & & & \\
\hline 4 & ¿Te ha parecido adecuado el material audiovisual utilizado fuera de las aulas? & & & \\
\hline 5 & $\begin{array}{l}\text { ¿Has echado de menos las explicaciones de la profesora durante las clases cuando algún } \\
\text { término no quedaba claro? }\end{array}$ & & & \\
\hline 6 & ¿Te has sentido bien trabajando en grupo para construir los propios aprendizajes? & & & \\
\hline 7 & ¿Te ha parecido adecuado variar la composición de los grupos para las diversas actividades? & & & \\
\hline 8 & $\begin{array}{l}\text { ¿Te parece adecuado trabajar las habilidades sociales y comunicativas a través de los } \\
\text { roleplay? }\end{array}$ & & & \\
\hline 9 & ¿Te has sentido cómodo durante los roleplay? & & & \\
\hline 10 & $\begin{array}{l}\text { ¿Has aprendido durante estas prácticas diversas técnicas y habilidades para la práctica clínica } \\
\text { con pacientes? }\end{array}$ & & & \\
\hline 11 & ¿Crees que la evaluación es adecuada para las competencias trabajadas? & & & \\
\hline
\end{tabular}

Indícame qué es lo que más te ha gustado.

Indícame que es lo que menos te ha gustado.

¿Tienes alguna sugerencia de mejora para años sucesivos? 